\title{
Two reaction channels directly observed for atomic hydrogen on the Si(111)-7×7 surface
}

\section{Citation}

Mortensen, K., D. M. Chen, P. J. Bedrossian, J. A. Golovchenko, and F. Besenbacher. 1991. Two Reaction Channels Directly Observed for Atomic Hydrogen on the Si(111)-7×7 Surface. Physical Review B 43, no. 2: 1816-1819. doi:10.1103/physrevb.43.1816.

\section{Published Version}

doi:10.1103/PhysRevB.43.1816

\section{Permanent link}

http://nrs.harvard.edu/urn-3:HUL.InstRepos:29407032

\section{Terms of Use}

This article was downloaded from Harvard University's DASH repository, and is made available under the terms and conditions applicable to Other Posted Material, as set forth at http:// nrs.harvard.edu/urn-3:HUL.InstRepos:dash.current.terms-of-use\#LAA

\section{Share Your Story}

The Harvard community has made this article openly available.

Please share how this access benefits you. Submit a story.

\section{Accessibility}




\title{
Two reaction channels directly observed for atomic hydrogen on the $\mathrm{Si}(111)-7 \times 7$ surface
}

\author{
K. Mortensen* \\ Institute of Physics, University of Aarhus, DK-8000 Aarhus C, Denmark; \\ Department of Physics, Harvard University, Cambridge, Massachusetts 02138; \\ and The Rowland Institute for Science, 100 Cambridge Parkway, Cambridge, Massachusetts 02142 \\ D. M. Chen, P. J. Bedrossian, ${ }^{\dagger}$ and J. A. Golovchenko \\ Department of Physics, Harvard University, Cambridge, Massachusetts 02138 \\ and The Rowland Institute for Science, 100 Cambridge Parkway, Cambridge, Massachusetts 02142 \\ F. Besenbacher \\ Institute of Physics, University of Aarhus, DK-8000 Aarhus C, Denmark \\ (Received 12 June 1990)
}

\begin{abstract}
Using scanning tunneling microscopy and spectroscopy, we have investigated the reaction of atomic hydrogen with the $\mathrm{Si}(111)-7 \times 7$ reconstructed surface. A simple picture evolves in which the reaction proceeds concurrently along two channels: a direct binding of the hydrogen atoms to the existing dangling bonds of the surface, and a slower removal of the uppermost Si layer and binding of the hydrogen atoms to the created dangling bonds of the next layer.
\end{abstract}

The interaction of hydrogen with silicon and in particular with silicon surfaces is of the utmost technological importance, partly because of the potential use of hydrogen to reduce the density of deep levels in both crystalline and amorphous $\mathrm{Si}$, and partly because of the use of silanes in the epitaxial growth of Si by chemical vapor deposition. The chemisorption of hydrogen on the $\mathrm{Si}(111)-7 \times 7$ reconstructed surface has been studied extensively for more than a decade. ${ }^{1-16}$ Although the structure of the clean $7 \times 7$ reconstruction is now generally accepted to be described by the dimer-adatom-stacking-fault model (the DAS model), ${ }^{17,18}$ the interaction of hydrogen with this surface is still open for vigorous dispute; e.g., does the DAS structure remain unchanged upon hydrogen exposure, ${ }^{9,12,16}$ or does an etching of the surface occur, ${ }^{6,10}$ and how can the large saturation coverage observed be explained ${ }^{5}$

Using the powerful capabilities of scanning tunneling microscopy (STM) and spectroscopy to probe both the surface topography and the electronic structure on an atomic scale, we report in the present paper the observation of two reaction channels for hydrogen on the $\mathrm{Si}(111)$ surface: (i) a direct binding of hydrogen to the existing surface dangling bonds, and (ii) a slower and limited removal of the topmost $\mathrm{Si}$ layer, whereby hydrogen is bound to the created dangling bonds of the next layer. This microscopic understanding facilitates a consistent explanation of the vast amount of previously published data.

The present experiments were carried out with a fully automated STM (Refs. 19 and 20) residing in an ultrahigh-vacuum chamber with a base pressure of $1 \times 10^{-8} \mathrm{~Pa}$. The Si crystal [(11), Sb doped to 0.01 $\Omega \mathrm{cm}$ ] were cleaned in situ by ion sputtering $\left(2-\mathrm{keV} \mathrm{Ne}^{+}\right)$ followed by annealing to $1300-1400 \mathrm{~K}$. This procedure resulted in a clean and defect-free $\mathrm{Si}(111)-7 \times 7$ surface as characterized by low-energy electron diffraction (LEED), Auger electron spectroscopy (AES), and scanning tunneling microscopy in combination. The surface was subsequently exposed at room temperature to a high-purity $\mathrm{H}_{2}$ gas (pressures ranging from $10^{-5}$ to $10^{-3} \mathrm{~Pa}$ ) dissociated at a hot tungsten filament (1800-1900 K). As the arrival rate of the atomic hydrogen was unknown we specify the dose of $\mathbf{H}_{2}$. To avoid appreciable heating of the sample due to radiation from the filament only short exposure times were employed and thus the sample temperature could be kept at 300-350 K. After adsorption of hydrogen the surface still displays a $7 \times 7$ LEED pattern, but with characteristic changes in the intensities of the fractional order spots, from which we obtain an estimate of the absolute hydrogen coverage. ${ }^{5}$ Contamination effects were effectively ruled out by the following controls: (i) with the filament turned on, but no inlet of hydrogen, the surface remained clean and free of defects as observed by STM, (ii) no contaminants were ever detected by AES, and (iii) desorption of the hydrogen at $900 \mathrm{~K}$ recreates an undisturbed $\mathrm{Si}(111)-7 \times 7$ surface as confirmed by STM, which rules out the presence of, e.g., oxygen and carbon, since these elements desorb at higher temperatures.

In Fig. 1 we show typical constant-current topographs for the clean surface (a), the surface exposed to $1.7 \times 10^{-2} \mathrm{~Pa}$ sec of hydrogen (b) and (c), and the surface exposed to $0.29 \mathrm{~Pa} \mathrm{sec}$ of hydrogen (d). The estimated coverages were $0.1-0.2 \quad \mathrm{ML} \quad[1 \quad$ monolayer $(\mathrm{ML})=7.8 \times 10^{14}$ atoms $\left./ \mathrm{cm}^{2}\right]$ for (b) and (c), and $0.8-0.1$ ML for (d). Since the tip height represented by the gray scale depends on both geometry and local electronic structure, care must be taken in the interpretation of such topographs. However, for the clean $\mathrm{Si}(111)-7 \times 7$ surface it is found that the bright spots in Fig. 1(a) do 

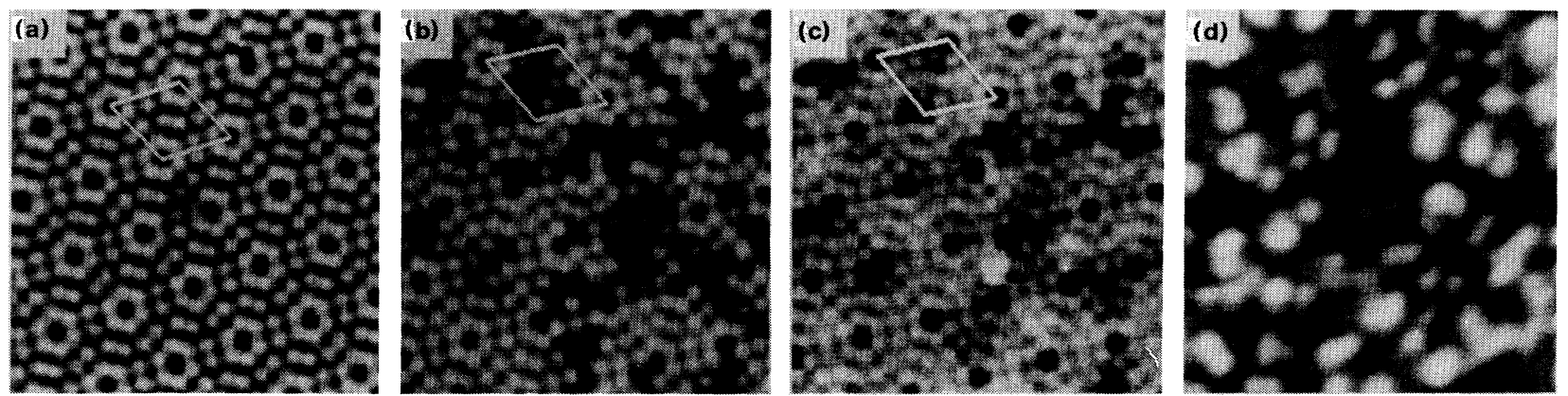

FIG. 1. Constant-current topographs of a $\mathrm{Si}(111)-7 \times 7$ surface of a $140 \times 140-\AA^{2}$ region. (a) Clean, $I_{t}=1.2 \mathrm{nA}, V_{s}=0.7 \mathrm{~V},(\mathrm{~b})$ and (c) after a low exposure to $\mathrm{H}_{2}\left(1.7 \times 10^{-2} \mathrm{~Pa}\right.$ sec), taken at the same location, with the same $7 \times 7$ unit cell outlined, but with $I_{t}=1.0$ $\mathrm{nA}, V_{s}=1.1 \mathrm{~V}$, and $I_{t}=1.2 \mathrm{nA}, V_{s}=2.5 \mathrm{~V}$, respectively, and (d) after a high exposure $\left(2.9 \times 10^{-1}\right.$ Pa sec $)$. The gray scale represents the tip height using white when the tip is retracted the most from the surface and vice versa, and the scales from black to white are 1 , 2,1 , and $3 \AA$, respectively.

indeed represent the $\mathrm{Si}$ atoms in the upper most layer, i.e., the adatoms. ${ }^{18}$

For the partially reacted surface we observe the following bias dependence similar to Ref. 16: at low bias voltages [Fig. 1(b)] a number of the $\mathrm{Si}$ adatoms apparently disappear, whereas at higher voltages [Fig. 1(c)] those atoms reappear. The disappearance at low bias and reappearance at high bias is independent of the bias polarity. This bias effect is attributed to the binding of a hydrogen atom at the adatom dangling bond, and we shall refer to it as the first reaction channel. The binding of hydrogen at the surface dangling bonds consequently eliminates the associated surface states near the Fermi level, and hence forces the probe tip closer to the surface to maintain a constant current at low bias voltages.

The interpretation given above is further substantiated by our local tunneling spectroscopy measurements. Figure 2 shows a set of averaged differential conductivity spectra, which are normalized in order to reflect the local density of states. ${ }^{21}$ For the clean surface [Fig. 2(a)] we observe the occupied states at $\approx 0.4 \mathrm{eV}$ and $\approx 1.0 \mathrm{eV}$ corresponding to the adatom dangling bonds and the dangling bonds in the next $\mathrm{Si}$ layer (at the so-called rest atoms), respectively, and the unoccupied state at $\approx 0.5 \mathrm{eV}$ related to the adatom dangling bonds. ${ }^{18,22}$ For a partially reacted surface $\left(2.9 \times 10^{-3} \mathrm{~Pa} \mathrm{sec}\right)$ an average over the reacted regions [Fig. 2(b)] shows a vanishing of both the adatom and the rest-atom dangling bond states, which manifests the binding of hydrogen to these existing dangling bonds. Moreover, an inspection of our nonaveraged tunneling spectra have shown no preferential reaction of either the adatom or the rest-atom dangling bonds, as opposed to the dissociative adsorption of ammonia at the $\mathrm{Si}(111)$ $7 \times 7$ surface. $^{23}$

As the reader may have noticed, not all the adatoms apparently absent in Fig. 1(b) reappear in Fig. 1(c). ${ }^{24}$ This second feature of adatom sites remaining dark at high bias voltages becomes much more pronounced at higher exposures [Fig. 1(d)]. A closer look at the dark regions is shown in Fig. 3. While the boundaries of the $7 \times 7$ unit cell are still present, the topographs now exhibit sublattices with a $1 \times 1$ periodicity. According to the DAS model this structure corresponds precisely to the

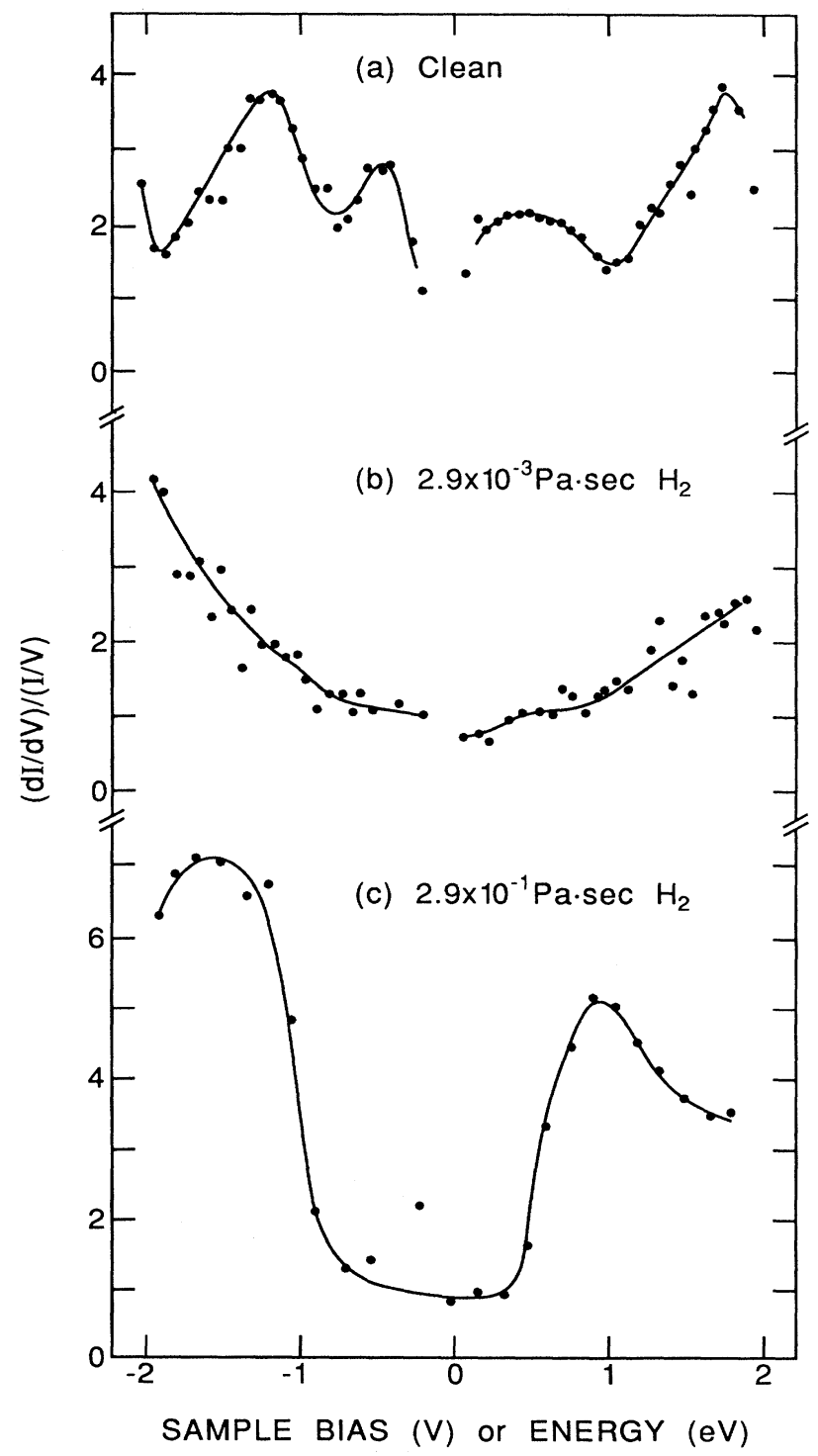

FIG. 2. Averaged normalized differential conductivity spectra for a $\mathrm{Si}(111)-7 \times 7$ surface. (a) Clean, (b) slightly $\mathrm{H}$ reacted $\left(2.9 \times 10^{-3} \mathrm{~Pa} \mathrm{sec}\right)$, and (c) highly $\mathrm{H}$ reacted $\left(2.9 \times 10^{-1} \mathrm{~Pa} \mathrm{sec}\right)$. 
stacking fault layer beneath the adatom layer. ${ }^{17}$ Note that even a saturation of all the adatom dangling bonds by hydrogen would not make the adatom layer "transparent" to the tunneling process at the high bias voltages used in Figs. 1(d) and 3. Rather, tunneling at high bias voltages will reveal the adatoms, just as in the case of Fig. 1(c). Thus, in contrast to the first reaction channel, the obtained tunneling images of the next Si layer (such as Fig. 3) cannot be accounted for merely by modifications of the electronic structure, but instead unambiguously demonstrate a second reaction, which involves a removal of the $\mathrm{Si}$ adatom layer.

Removing the adatoms will leave a surface with 43 dangling bonds for each $7 \times 7$ unit cell. These dangling bonds are expected to give rise to a high density of states at or near the Fermi level. However, the tunneling spectra obtained in these regions [Fig. 2(c)] exhibit a band gap of $\approx 1.2 \mathrm{eV}$, which indicates that all the dangling bonds are terminated by hydrogen atoms as a part of the reaction.

Since a continued removal of $\mathrm{Si}$ atoms was never observed in the present studies after hydrogen exposure was terminated, the binding of hydrogen at an existing dangling bond is inferred to be a stable state at RT and not a precursor for the etching process. Consequently, the second reaction must proceed directly from hydrogen in the gas phase, and we shall refer to it as the second channel for the reaction of hydrogen with $\mathrm{Si}(111)-7 \times 7$.

Considering the dynamics of the second reaction channel, we first note that the bond energy for a $\mathrm{Si}-\mathrm{H}$ bond is higher than that of a $\mathrm{Si}-\mathrm{Si}$ bond by more than 1 eV ${ }^{6,13,25}$ Hence the breaking of a Si-Si bond followed by the formation of $\mathrm{Si}-\mathrm{H}$ bonds is an energetically favorable process, even if only one of the two created dangling bonds captures a hydrogen atom. However, as inferred from the observed slow adatom depletion rate, the process of breaking the three backbonds of each $\mathrm{Si}$ adatom one by one in favor of $\mathrm{Si}-\mathrm{H}$ bonds is limited by a considerable activation barrier. Secondly, the inspection of topographs for a wide range of exposures has not revealed

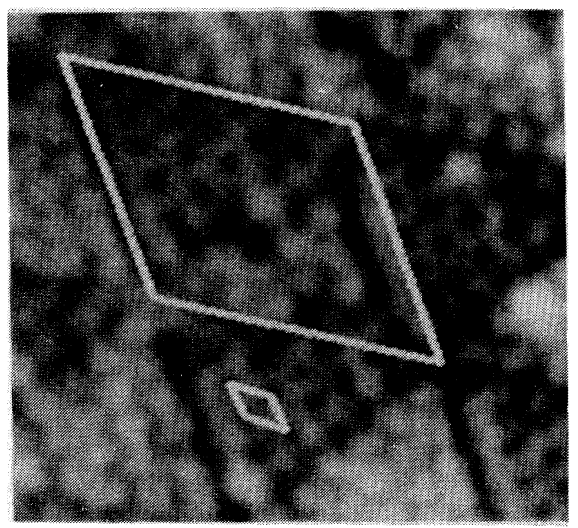

FIG. 3. High-resolution topograph of a highly $\mathbf{H}$ reacted Si(111)-7×7 surface $\left(2.9 \times 10^{-1} \mathrm{~Pa} \mathrm{sec}\right) . \quad 55 \times 55 \AA^{2}, I_{t}=2.4$ na, $V_{s}=1.8 \mathrm{~V}$. Both a $7 \times 7$ and a $1 \times 1$ unit cell are outlined. The height scale is $1.5 \AA$. any preferential removal of adatoms at different atomic sites, implying that there is no substantial local variation in the activation barrier. Thirdly, from the continuous observation of stacking-fault regions in the STM topographs, we can also conclude that a significant removal of $\mathrm{Si}$ atoms in the next layer does not occur. This limitation of the etching process indicates a much higher activation barrier for breaking of $\mathrm{Si}-\mathrm{Si}$ bonds in the next layer. We suggest that the difference in the activation barrier originates from the different bonding configurations with the adatoms having highly strained bond angles, which results in a weaker binding compared to $\mathrm{Si}$ atoms in the next layer.

The present findings directly establish the existence of two distinct reaction channels for the atomic hydrogen interaction with the $\mathrm{Si}(111)-7 \times 7$ surface. In light of this microscopic understanding, much of the previous data can now be explained consistently.

First, the persistence of the $7 \times 7$ symmetry up to a saturation coverage as observed by LEED (Refs. 2, 4, and 5) is explained by the preservation of the stacking fault upon the removal of the $\mathrm{Si}$ adatom layer, and it also supports the suggested limitation of the etching to the adatom layer. In addition, the characteristic changes in the LEED intensities upon saturation can be explained qualitatively by adapting the kinematical analysis of McRae and Caldwell ${ }^{4}$ to the present picture.

The etching observed here has also been reported in another STM investigation. ${ }^{26}$ It is consistent with the detection of $\mathrm{SiH}_{3}$ and $\mathrm{SiH}_{4}$ in the mass spectrometer during $\mathrm{H}$ exposure, ${ }^{6,10}$ and provides direct evidence for the interpretation of the very recent core-level shift and photoemission spectroscopy measurements. ${ }^{15}$ The removal of $\mathrm{Si}$ adatoms also explains the residual disruption of the original $7 \times 7$ reconstruction observed in both LEED (Refs. 6, 8, and 9) and the present STM studies even after a complete desorption of hydrogen at $750 \mathrm{~K}$. In fact, a perfect $7 \times 7$ STM image [similar to Fig. 1(a)] is obtained only after annealing at higher temperatures (900 K). Therefore, once the etching takes place in the first adsorption, subsequent cycles of desorption (at modest temperatures) and adsorption will not further induce substantial changes of the Si geometry, since at modest temperatures the removed $\mathrm{Si}$ adatom layer cannot be recreated by surface diffusion. Thus, unlike what was previously argued, the fact that repetitive adsorption and desorption cause no changes in photoemission spectra ${ }^{12}$ or in electron energy-loss spectra ${ }^{9}$ does not conflict with the etching behavior.

Since the removal of the adatoms leaves no $\mathrm{Si}$ atoms with more than one dangling bond, the resulting surface cannot accommodate the additional dihydride and/or trihydride species, which are observed at high hydrogen coverages by both electron energy-loss and infrared spectroscopies. $^{7-9}$ We emphasize, however, that an exceedingly high exposure is required to remove all the $\mathrm{Si}$ adatoms and obtain a true saturation. We thus propose that, in addition to the atoms at steps and defects, $7,12,14$ remaining $\mathrm{Si}$ adatoms are responsible for the formation of monohydride, dihydride, or trihydride species depending on the number of broken backbonds. Accordingly, the 
white areas in Fig. 1(d) could be attributed to such remaining adatoms. A similar model with trihydride species randomly distributed on the next $\mathrm{Si}$ layer is reported to give a better agreement with measurements of reflection high-energy electron-diffraction intensities. ${ }^{11}$

Finally, a saturation coverage of $1.25 \pm 0.13 \mathrm{ML}$ has been measured previously by nuclear-reaction analysis ${ }^{5}$ (NRA) whereas a complete removal of the adatoms and saturation of the resulting dangling bonds only gives a coverage of $\frac{43}{49}=0.88 \mathrm{ML}$. Although this discrepancy could be reconciled by considering the formation of dihydride and trihydride species with some remaining $\mathrm{Si}$ adatoms, it would need $\frac{3}{4}$ or more of the adatoms to remain at the surface to account for the measured coverage, which is inconsistent with the present observations. Alternatively, we suggest as a probable process the breaking of the strained dimer bonds and formation of additional monohydride species at the created free bonds. In this case the saturation amounts to $\frac{61}{49}=1.24 \mathrm{ML}$, which then explains the NRA result stated above.

In summary, our atomic-scale observations of the interaction of atomic hydrogen with the $\mathrm{Si}(111)-7 \times 7$ surface have revealed two different reaction channels: a direct binding of hydrogen to the existing surface dangling bonds, and a slower and limited etching of Si atoms and binding of hydrogen atoms to the created dangling bonds of the next layer. This microscopic observation unifies many earlier studies and sheds new light on the understanding of the interaction of atomic hydrogen with silicon and its technological applications.

We gratefully acknowledge F. Jensen, E. Lægsgaard, and I. Stensgaard for valuable cooperation and discussion. This work was supported by the Danish Research Council through "Center for Surface Reactivity," the Kund Højgaard Foundation, and the U.S. Office of Naval Research (Contract No. N00014-87-K-0511). K. M. also acknowledges the financial support of NATO.
${ }^{*}$ Present address: Department of Applied Science, Bldg. 480, Brookhaven National Laboratory, Upton, NY 11973.

Present address: Division 1111, Sandia National Laboratories, P. O. Box 5800, Albuquerque, NM 87185-5800.

${ }^{1}$ H. Ibach and J. E. Rowe, Surf. Sci. 43, 481 (1974).

${ }^{2}$ T. Sakurai and H. D. Hagstrum, Phys. Rev. B 12, 5349 (1975); 14, 1593 (1976).

${ }^{3}$ J. A. Appelbaum and D. R. Hamann, Phys. Rev. Lett. 34, 806 (1975).

${ }^{4}$ E. G. McRae and C. W. Caldwell, Phys. Rev. Lett. 46, 1632 (1981).

${ }^{5}$ R. J. Culbertson, L. C. Feldman, P. J. Silverman, and R. Haight, J. Vac. Sci. Technol. 20, 868 (1982).

${ }^{6}$ G. Schulze and M. Henzler, Surf. Sci. 124, 336 (1983).

${ }^{7}$ Y. J. Chabal, G. S. Higashi, and S. B. Christman, Phys. Rev. B 28, 4472 (1983).

${ }^{8}$ R. Butz, E. M. Oellig, H. Ibach, and H. Wagner, Surf. Sci. 147, 343 (1984).

${ }^{9}$ H. Froitzheim, U. Köhler, and H. Lammering, Surf. Sci. 149, 537 (1985).

${ }^{10}$ D. R. Olander, M. Balooch, J. Abrefah, and W. J. Siekhaus, J. Vac. Sci. Technol. B 5, 1404 (1987).

${ }^{11}$ A. Ichimiya and S. Mizuno, Surf. Sci. 191, L765 (1987).

${ }^{12}$ D. Muller, F. Ringeisen, J. J. Koulman, and D. Bolmont, Surf. Sci. 189/190, 472 (1987).

${ }^{13}$ B. G. Koehler, C. H. Mak, D. A. Arthur, P. A. Coon, and S. M. George, J. Chem. Phys. 89, 1709 (1988).

${ }^{14}$ R. Imbihl, J. E. Demuth, S. M. Gates, and B. A. Scott, Phys. Rev. B 39, 5222 (1989).
${ }^{15}$ C. J. Karlsson, E. Landemark, L. S. O. Johansson, U. O. Karlsson, and R. I. G. Uhrberg, Phys. Rev. B 41, 1521 (1990).

${ }^{16}$ T. Sakurai, Y. Hasegawa, T. Hashizume, I. Kamiya, T. Ide, I. Sumita, H. W. Pickering, and S. Hyodo, J. Vac. Sci. Technol. A 8, 259 (1990).

${ }^{17} \mathrm{~K}$. Takanayagi, Y. Tanishiro, M. Takahashi, and $\mathrm{S}$. Takahashi, J. Vac. Sci. Technol. A 3, 1502 (1985).

${ }^{18}$ R. M. Tromp, R. J. Hamers, and J. E. Demuth, Science 234, 304 (1986).

${ }^{19}$ E. Lægsgaard, F. Besenbacher, K. Mortensen, and I. Stensgaard, J. Microsc. 152, 663 (1988).

${ }^{20}$ Preliminary measurements were performed at Harvard University and The Rowland Institute for Science with similar results, see K. Mortensen, D. M. Chen, P. J. Bedrossian, G. Maalouf, and J. A. Golovchenko, Bull. Am. Phys. Soc. 34, 820 (1989).

${ }^{21}$ J. A. Stroscio, R. M. Feenstra, and A. P. Fein, Phys. Lett. 57, 2579 (1986)

${ }^{22}$ R. I. G. Uhrberg, G. V. Hansson, J. M. Nicholls, P. E. S. Persson, and S. A. Flodström, Phys. Rev. B 31, 3805 (1985); J. M. Nicholls and B. Reihl, ibid. 36, 8071 (1987).

${ }^{23} \mathrm{Ph}$. Avouris and R. Wolkow, Phys. Rev. B 39, 5091 (1989).

${ }^{24}$ This can also be seen in Fig. 4 of Ref. 16, but since only low coverages were employed in Ref. 16, this effect was not noticed.

${ }^{25}$ For example, in T. L. Cottrell, The Strength of Chemical Bonds (Butterworths, London, 1954).

${ }^{26}$ J. J. Boland, Bull. Am. Phys. Soc. 35, 226 (1990), and private communication. 

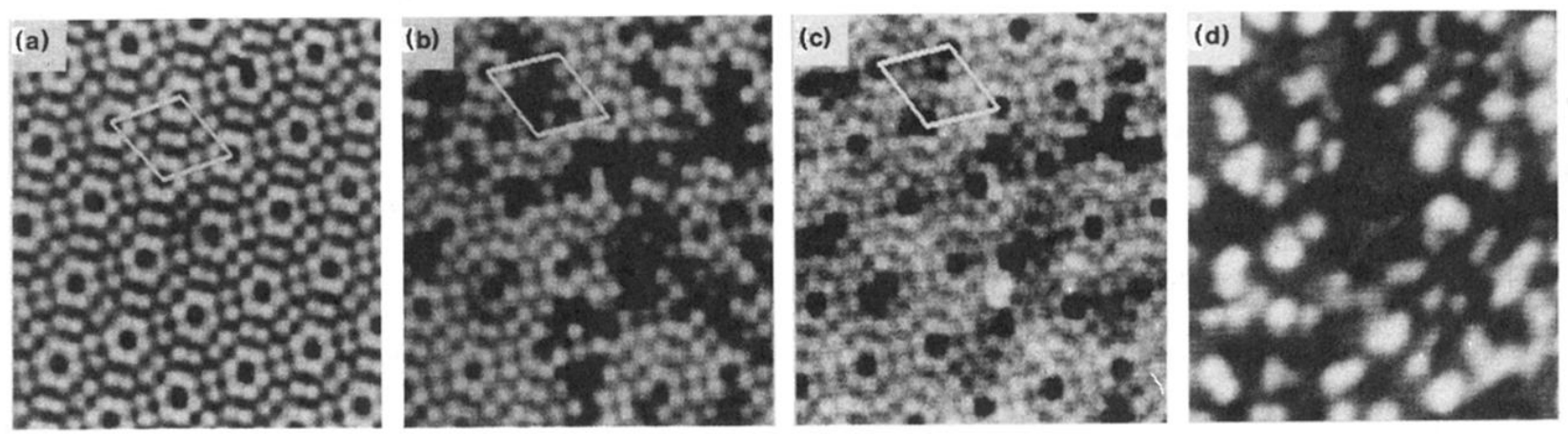

FIG. 1. Constant-current topographs of a Si(111)-7 $\times 7$ surface of a $140 \times 140-\AA^{2}$ region. (a) Clean, $I_{t}=1.2 \mathrm{nA}, V_{s}=0.7 \mathrm{~V},(\mathrm{~b})$ and (c) after a low exposure to $\mathrm{H}_{2}\left(1.7 \times 10^{-2} \mathrm{Pasec}\right)$, taken at the same location, with the same $7 \times 7$ unit cell outlined, but with $I_{t}=1.0$ $\mathrm{nA}, V_{s}=1.1 \mathrm{~V}$, and $I_{t}=1.2 \mathrm{nA}, V_{s}=2.5 \mathrm{~V}$, respectively, and (d) after a high exposure $\left(2.9 \times 10^{-1}\right.$ Pa sec). The gray scale represents the tip height using white when the tip is retracted the most from the surface and vice versa, and the scales from black to white are 1 , 2,1 , and $3 \AA$, respectively. 


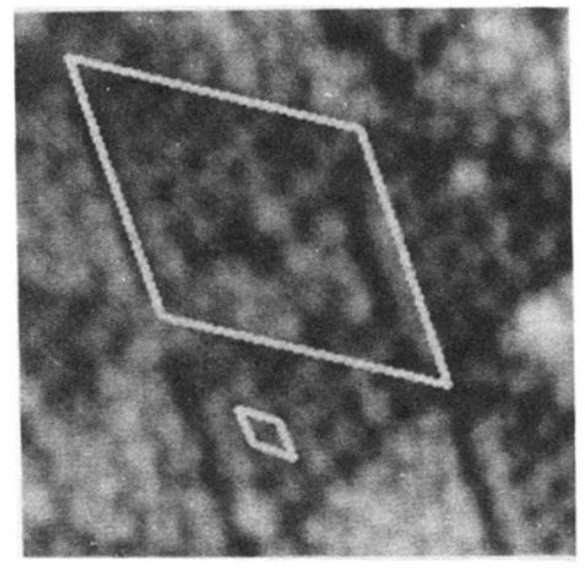

FIG. 3. High-resolution topograph of a highly $\mathrm{H}$ reacted Si(111)-7 $7 \times 7$ surface $\left(2.9 \times 10^{-1}\right.$ Pa sec). $55 \times 55 \AA^{2}, I_{t}=2.4$ na, $V_{s}=1.8 \mathrm{~V}$. Both a $7 \times 7$ and a $1 \times 1$ unit cell are outlined. The height scale is $1.5 \AA$. 